\title{
Inducing circular vection with tactile stimulation encircling the waist
}

\author{
Angelica M. Tinga ${ }^{a, b, c, *}$, Chris Jansen ${ }^{\mathrm{a}}$, Maarten J. van der Smagt ${ }^{\mathrm{b}}$, Tanja C.W. Nijboer ${ }^{\mathrm{b}, \mathrm{d}}$, \\ Jan B.F. van $\operatorname{Erp}^{\mathrm{a} \text {, }}$ \\ a TNO, Department of Perceptual and Cognitive Systems, Soesterberg, The Netherlands \\ b Utrecht University, Department of Experimental Psychology, Helmholtz Institute, Utrecht, The Netherlands \\ ${ }^{\mathrm{c}}$ Tilburg University, Department of Communication and Information Sciences, Tilburg, The Netherlands \\ d Brain Center Rudolf Magnus, and Center of Excellence for Rehabilitation Medicine, University Medical Center Utrecht, and De Hoogstraat Rehabilitation, The Netherlands \\ e Twente University, Department of Human Media Interaction, Enschede, The Netherlands
}

\section{A R T I C L E I N F O}

\section{Keywords:}

Vection

Illusory self-motion

Tactile stimulation

Walking

Balance

Sway

\begin{abstract}
A B S T R A C T
In general, moving sensory stimuli (visual and auditory) can induce illusory sensations of self-motion (i.e. vection) in the direction opposite of the sensory stimulation. The aim of the current study was to examine whether tactile stimulation encircling the waist could induce circular vection (around the body's yaw axis) and to examine whether this type of stimulation would influence participants' walking trajectory and balance. We assessed the strength and direction of perceived self-motion while vision was blocked and while either receiving tactile stimulation encircling the waist clockwise or counterclockwise or no tactile stimulation. Additionally, we assessed participants' walking trajectory and balance while receiving these different stimulations. Tactile stimulation encircling the waist was found to lead to self-reported circular vection in a subset of participants. In this subset of participants, circular vection was on average experienced in the same direction as the tactile stimulation. Additionally, perceived rotatory self-motion in participants that reported circular vection correlated with balance (i.e., sway velocity and the standard error of the mean in the medio-lateral dimension). The fact that, in this subset of participants, subjective reports of vection correlated with objective outcome measures indicates that tactile stimulation encircling the waist might indeed be able to induced circular vection.
\end{abstract}

\section{PsycINFO classification}

2300 Human Experimental Psychology 2320 Sensory Perception

\section{Introduction}

An illusory experience of self-motion (i.e. vection) can be induced by moving stimuli, even in absence of physical movement of the body (e.g. Lestienne, Soechting, and Berthoz, 1977; Riecke, Feuereissen, and Rieser, 2008). Several slightly different definitions of vection exist. In this article vection is defined as the sensation of self-motion induced by moving sensory stimulation not corresponding to the veridical selfmotion. Self-motion illusions occurring in a linear fashion (i.e. translation along one or more of the three body axes) are referred to as linear vection. The illusion of rotation about one or more of the three body axes is referred to as circular vection (Väljamäe, 2009). In general, vection is experienced in the direction opposite to the sensory stimulation (Andersen, 1986; Riecke, Väljamäe, and Schulte-Pelkum, 2009;
Väljamäe, 2009). However, a few studies have demonstrated that vection can be experienced in the same direction as the sensory stimulation (Nakamura \& Shimojo, 2000 and 2003; Seno, Ito, and Sunaga, 2009).

Vection can be induced by stimulation in different (combinations of) sensory modalities. Visually-induced vection is the most studied type, with visual stimulation being able to induce both linear and circular vection (Andersen, 1986). Visually-induced vection can be modified by vestibular stimulation (Lepecq et al., 2006). Vestibular stimulation by itself (through electrical stimulation of the vestibular system) can also induce vection, with longer stimulation (at least $400 \mathrm{~ms}$ ) and with higher currents (when tested with currents ranging from 0.5-4 mA) being more likely to induce an illusion of continuous movement (Fitzpatrick, Burke, and Gandevia, 1994; Wardman, Taylor, and Fitzpatrick, 2003). Auditory stimulation can enhance visually-induced vection as well (Riecke et al., 2009) and it can induce linear and circular vection by itself (Väljamäe, 2005 and 2009).

In addition to the subjective reports of vection, vection can influence the spatial reference frame as for example reflected in its influence on balance and walking. These effects are often interpreted as a

\footnotetext{
* Corresponding author at: Tilburg University, Dante Building Room D351, Warandelaan 2, 5037 AB Tilburg, The Netherlands.

E-mail address: A.M.Tinga@uvt.nl (A.M. Tinga).
} 
correction to compensate for the perceived self-motion (e.g. Fitzpatrick et al., 1994; Wardman et al., 2003). In general, visually-induced linear and circular vection induce body displacements in the same direction as that of the moving visual stimulus (Bronstein and Buckwell, 1997; Fushiki, Kobayashi, Asai, and Watanabe, 2005; Kapteyn and Bles, 1977; Reason, Wagner, and Dewhurst, 1981) and when movement of the visual stimulus stops, participants return to an upright position and there after lean in the opposite direction (Reason et al., 1981). However, participants may report vection without a balance shift, or change their balance without reporting vection (Guerraz and Bronstein, 2008) or before vection is reported (Fushiki et al., 2005). Moving sounds from side to side or rotating around the participant's head induce vection and elicit lateral sway (Al'tman, Varyagina, and Levik, 2005; Soames and Raper, 1992; Tanaka, Kojima, Takeda, Ino, and Ifukube, 2001), yet not in a systematic direction. Regarding stimulation of the vestibular system, postural and locomotor deviations toward the stimulated side have been reported (Fitzpatrick et al., 1994; Bent, McFadyen, French Merkley, Kennedy, and Inglis, 2000; Cauquil, Martinez, Ouaknine, and Tardy-Gervet, 2000).

Thus, there is considerable evidence for vection induced by auditory, vestibular, and especially visual stimulation. Yet, research on tactile stimulation and vection is rather scarce and has generally focused on whether tactile stimulation can facilitate vection induced by stimulation of another sensory modality. For example, the addition of vibrations on an area of the body can enhance both visually-induced linear and circular vection (Riecke, Schulte-Pelkum, Caniard, and Bulthoff, 2005) and auditorily induced linear (Väljamäe, 2005) and circular (Riecke et al., 2008) vection. However, inhibition of vection by tactile stimulation has also been reported in a few participants (Riecke et al., 2005). Additionally, self-motion illusions induced by non-moving tactile stimulation on the supporting areas of the feet in standing participants are reported in three studies (Nilsson, Nordahl, Sikström, Turchet, and Serafin, 2012; Nordahl, Nilsson, Turchet, and Serafin, 2012; Roll, Kavounoudias, and Roll, 2002). Roll et al. (2002) first reported that ten seconds of stimulation on the supporting areas of both feet could induce illusions of linear self-motion (orthogonally directed and ipsilateral to the vibrated area of the feet) in 7 out of 10 blindfolded and restrained (to prevent real movement) participants. Nordahl et al. (2012) and Nilsson et al. (2012) continued this work by presenting participants different virtual environments (an elevator (Nordahl et al., 2012) or an elevator, train, bathroom, and darkness (Nilsson et al., 2012)). Identical tactile stimulation on the supporting areas of both feet could induce horizontal and vertical illusory linear self-motion, depending on the virtual environment. Notably, all studies examining the effect of tactile stimulation on the illusion of self-motion did not present moving tactile stimulation but rather examined whether tactile stimulation can induce uncertainty to the vestibular system and therefore increase the weighting of signals of other sensory modalities or whether it can increase the convincingness of motion simulation. Therefore, to our knowledge, vection induced predominantly by moving tactile stimulation and its effects on walking and balance have not been reported yet. The role of visual, vestibular and auditory information in determining (illusory) self-motion might appear more straightforward than the role of tactile information. Yet, tactile cues appear to play an important role in determining self-motion as well, as for example, air that flows over the skin during movement appears to play an important role in determining self-motion (Seno, Ogawa, Ito, and Sunaga, 2011). Additionally, tactile cues can influence orientation, especially when more weight is given to these cues compared to other sensory cues (van Erp and van Veen, 2006). Therefore, tactile-induced vection might be expected to influence walking and balance.

In earlier studies in our lab (Bos, van Erp, Groen, and van Veen, 2005; van Erp, Groen, Bos, and van Veen, 2006) several participants anecdotally reported circular vection as a result of receiving tactile stimulation encircling the torso. In these experiments, densely spaced vibrating elements were used to create a sensation of smooth apparent motion. However, these studies did not systematically measure circular vection. The aim of the current study was to (1) verify whether comparable tactile stimulation encircling the waist could induce circular vection around the body's yaw axis and (2) examine whether this type of stimulation would influence walking and balance. To this end, we assessed participants' subjective strength and direction of perceived self-motion while their vision was blocked and while they received tactile stimulation encircling the waist clockwise or counterclockwise or no tactile stimulation. Additionally, we assessed participants' walking trajectory and balance while their vision was blocked and while receiving these different stimulations.

It was expected that participants would experience clockwise circular vection with counterclockwise tactile stimulation and counterclockwise circular vection with clockwise tactile stimulation. In addition, it was expected that tactile stimulation would lead to the participants' walking trajectory and balance to be shifted in the same direction as the tactile stimulation. Specifically, participants' walking trajectory was expected to deviate and participants' balance was expected to shift to the right with clockwise and to the left with counterclockwise tactile stimulation.

\section{Method}

\subsection{Participants}

A total of 40 participants gave written and verbal consent and participated in this study, 20 female and 20 male. The participants were aged in between 40 and 60 years (Mean $=52.30 \pm S D=6.13$ ). The criteria for exclusion were: (1) history of orthopedic disorders; (2) usage of medication that is known to influence the vestibular system; (3) usage of assistive devices for standing; and (4) not being able to stand in the Romberg position (an upright position with legs stretched, feet together and arms held next to the body) with the eyes closed for $30 \mathrm{~s}$ (assessed when participants arrived in the lab). Participants received 30 euros for their participation. The study was approved by the TNO Institutional Review Board (Ethical Application Ref: TNO-IRB2013-12-31) and was conducted according to the principles expressed in the Declaration of Helsinki.

\subsection{Apparatus, stimuli and measures}

\subsubsection{Tactile stimulation}

Tactile stimulation was presented by means of a 'belt' consisting of a string of 13 vibration elements (i.e. tactors) mounted on elastic textile, developed by Elitac, Amsterdam, the Netherlands. This belt was worn around the waist at approximately $6 \mathrm{~cm}$ above the participant's navel over one layer of thin clothing. The tactors were lightly pressed on the skin by the elastic textile. The tactors had a contact area of 28 by $9 \mathrm{~mm}$ and generated a $158 \mathrm{~Hz}$ oscillation. The optimal temporal parameters for the tactile stimulation were determined in a pilot study in which 10 research interns of TNO Human Factors participated. Participants in the pilot study indicated for 6 different stimulations how strongly they experienced self-rotation while they were seated with their eyes closed. A sequential oscillation of each tactor for 308 milliseconds with an overlap of 154 milliseconds elicited the strongest self-reported circular vection in the pilot study and these parameters were used in the current study. In this way the vibration travelled the whole waist (clockwise or counterclockwise) in about $2 \mathrm{~s}$. This stimulation elicited weak self-reported circular vection $(M=3, S D=1.78$, on a scale from 1 to 10 , ranging from 'not strong at all' to 'absolutely strong') in the pilot study and is within the range of optimal tactile apparent motion (van Erp, 2007). The tactile stimulation was demonstrated before starting the experiment. The expected effects of the tactile stimulation on the study's outcome measures were not disclosed at any time during the study. 


\subsubsection{Cognitive load}

To ensure that walking and balance predominantly relied on automatic processes, cognitive load was induced with an auditory 2-back task presented via wireless headphones (Plantronics Pulsar 590, custom made). To reduce external sound influences, the headphones were integrated in acoustic earmuffs and pink noise was played in the background of the task. The cognitive load task consisted of a sequence in which eight different spoken letters were presented randomly in a succession of about $3.1 \mathrm{~s}$. Twenty-five percent of the spoken letters were targets (i.e. the same letter as two letters earlier). Manual responses for both targets and non-targets were given via a wireless presenter (Keningston SI600). Two seconds after presentation of the letter, a sound (of $<1 \mathrm{~s}$ ) was presented: a sound with high tones when a correct response was given, a 'fail buzzer' when a wrong response or no response was given. The task was created and played using MATLAB version 7.5 and Psychophysics Toolbox version 3.0.11.

\subsubsection{Walking trajectory}

A LIDAR (SICK LMS 100-10,000, custom made) was used to record participants' walking trajectory. The SICK LIDAR had a field of view of $180^{\circ}$ and an angular resolution of $0.25^{\circ}$. It scanned with a frequency of $50 \mathrm{~Hz}$ in a sensing range up to $13 \mathrm{~m}$. The LIDAR was placed at an altitude of about $44 \mathrm{~cm}$ above the ground with the scanning plane parallel to the floor to obtain information from the participant's legs without detecting the feet. The position of the participants was calculated as the mean of the data points that the legs of the participants provided. The total area in which participants could walk was about $12 \times 14 \mathrm{~m}$. The point at which participants started walking was positioned between two sturdy cardboard boxes, which were about $94 \mathrm{~cm}$ high. The participants started walking in-between the boxes for about $82 \mathrm{~cm}$, this would lead participants to set their first steps approximately in the 'straight-ahead direction'.

\subsubsection{Balance}

Balance was measured with a Nintendo Wii Balance Board of approximately $32 \mathrm{~cm} \times 51 \mathrm{~cm} \times 5 \mathrm{~cm}$. The communication between the Balance Board and a MSI notebook (U100) was established via Bluetooth. Data was sampled at about $16 \mathrm{~Hz}$.

\subsubsection{Self-reported circular vection}

The measurement of the subjective intensity of circular vection was based on a measurement of subjective linear vection from Wright, DiZio, and Lackner (2006). In the current study, the sensation of selfrotation was assessed with two different questions (as opposed to one in Wright's study (Wright et al., 2006)), namely: 1) "How strongly do you perceive that you are rotating?"; and 2) "How strongly do you perceive that you are rotating with reference to the external environment as opposed to perceiving something moving around your body?". The questions had to be rated on a scale from 0 to 5 where ' 0 ' represented "no perceived rotatory self-motion" or "movement around the body" and ' 5 ' "fully compelling rotatory self-motion" or "self-rotation with reference to the external environment" for the first and second question respectively (in Wright's study (Wright et al., 2006) a scale from 0 to 5 was used to measure subjective linear vection as opposed to subjective circular vection).

\subsection{Task and procedure}

Participants first performed the task in which their walking trajectory was assessed, followed by the task in which their balance was assessed. Subjective perceived self-motion was assessed at the end of the study in order to prevent participants from having any insights in the expected effects of the tactile stimulation. The whole testing session lasted approximately $1.5 \mathrm{~h}$ per participant.

Before starting data collection, participants put on a pair of slippers and stood in the Romberg position for $30 \mathrm{~s}$ while their vision (including

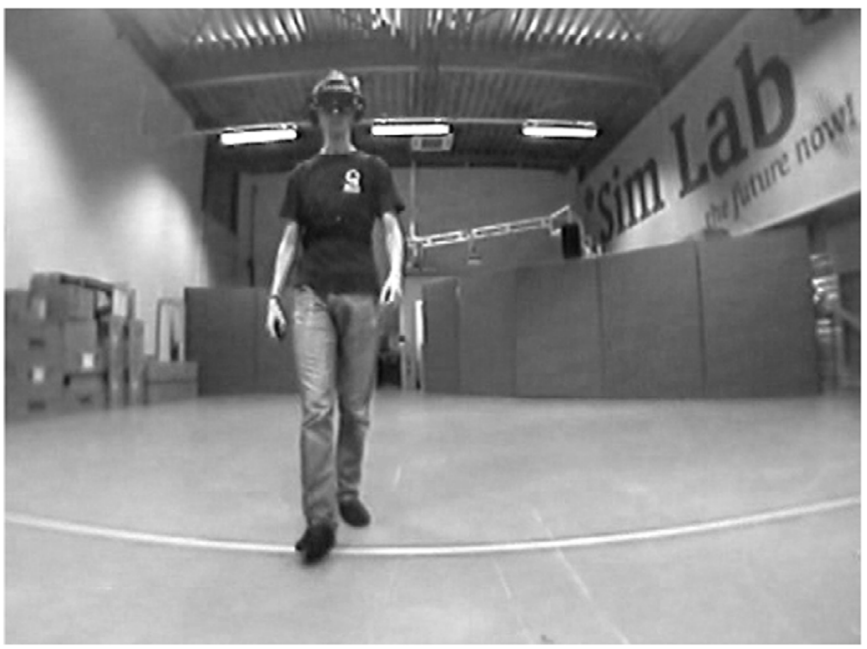

Fig. 1. In the walking task, participants walked straight ahead while wearing non-seethrough glasses and acoustic earmuffs. Responses on the cognitive load task were given via a wireless presenter (right hand). When the sound of the cognitive load task stopped after about $10 \mathrm{~m}$, participants stopped walking. While walking, either tactile stimulation encircling the waist clockwise or counterclockwise or no stimulation was presented.

central and peripheral vision) was blocked with non-see-through glasses. Then, participants received instructions on the cognitive load task. Each letter required a response; either the right or left button of the wireless presenter had to be pressed after the presentation of a target letter or a non-target letter respectively. Making no mistakes was emphasized to be very important. Participants practiced until performance was at least $80 \%$ after having performed at least 10 practice trials. This $80 \%$ criterion was only applied during practice of the cognitive load task. Next, the tactile belt was placed tightly around the waist of the participants and the tactile stimulation was demonstrated.

\subsubsection{Walking task}

Participants were instructed to walk straight ahead while their vision was blocked by the glasses and while performing the cognitive load task (Fig. 1). They were free to choose their walking speed and were instructed not to speak and to make no sounds while walking.

After one practice trial, data collection in the walking task started. In each trial, participants started in a fixed starting position with their feet about $20 \mathrm{~cm}$ apart. The experimenter indicated whether a vibration would be felt in the upcoming trial and instructed the participants to put the non-see-through glasses on and get ready. When the participants indicated they were ready, the cognitive load task was started. After presentation of two letters of the load task, the word 'start' was presented over the headphones and the participants started walking. They first walked in between the cardboard boxes while they let their hands slide over the boxes' edges. After walking about $10 \mathrm{~m}$, or if participants were too close to the walls of the testing room, the cognitive load task was stopped and the participants stopped walking. The experimenter led the participants back to the starting point in a fixed zigzag course. Approximately $1 \mathrm{~m}$ in front of the starting position participants took off the glasses and got back in the right position for the next trial. This was repeated 15 times. Tactile stimulation was given in a random order in 10 out of 15 trials, of which 5 stimulations were clockwise and 5 counterclockwise. Stimulation was started when participants started walking and stopped before walking back to the starting point.

\subsubsection{Balance task}

Participants were instructed to perform the cognitive load task while standing in the Romberg position. Data collection was started after one practice trial. In each trial, the experimenter first indicated whether a vibration would be felt in the upcoming trial. Next, 
participants took place in the right position on the Balance Board. Then, the experimenter gave instructions to put the non-see-through glasses on and to get ready. When the participants were ready, the cognitive load task was started. When the sound of the cognitive load task stopped, participants took off the glasses and stepped off the Balance Board. Data sampling started when the cognitive load task was started and continued for about $37 \mathrm{~s}$. This was repeated 12 times. Tactile stimulation was given in a random order in 8 of the 12 trials, of which 4 stimulations were clockwise and 4 counterclockwise. Stimulation was started after about $14 \mathrm{~s}$ after the start of the cognitive load task.

\subsubsection{Subjective task}

To help participants quantify the subjective measure, two examples were given in the written instructions. With the train illusion example (i.e. experiencing momentary illusory self-motion while sitting in a stationary train when a train on an adjacent track pulls away) the meaning of illusory self-motion was introduced. To give an example of illusory rotatory self-motion, the after-effect occurring when just being rotated on a desk chair was described. Participants were instructed to answer ' 0 ' on the estimation scale when experiencing no sensation of rotatory self-motion and to answer ' 5 ' when experiencing a high sensation of rotatory self-motion which is compelling and in a clear direction. After the instructions, participants stood in the Romberg Position. When ready, participants put on the non-see-through glasses and tactile stimulation or no stimulation started. After about $10 \mathrm{~s}$, both questions of the measurement of the subjective intensity of circular vection were asked by the experimenter to which the participants verbally responded. When participants answered ' 1 ' or higher on a question, they were asked to indicate the direction in which they experienced the rotation (clockwise or counterclockwise). After answering both questions, the stimulation stopped and participants took the non-see-through glasses off and looked around for about $10 \mathrm{~s}$. This was repeated three times. Once with clockwise, once with counterclockwise, and once with no tactile stimulation, in a random order.

\subsection{Data analysis}

In this section we will first discuss the analyses of the subjective task, followed by the analyses of the walking and balance tasks. Nonparametric statistical tests were used for all tests, as data significantly deviated from a normal distribution as shown by Kolmogorov-Smirnov tests, with $D(40)$ reaching $0.43(p<0.001), D(39)$ reaching 0.20 $(p<0.001)$ and $D(33)$ reaching $0.21(p<0.001)$ for the subjective, walking and balance data respectively.

\subsubsection{Subjective task}

First, we statistically examined the effect of rotatory tactile stimulation on the strength of self-reported circular vection (irrespective of the indicated direction). Data on the first question were analyzed with a Friedman test and a Kendall's coefficient of concordance $(W)$ test for computing the level of agreement between subjects ranging from 0 (no agreement) to 1 (complete agreement (Field, 2009)), and post-hoc multiple comparisons following the stepwise step-down method. Additionally, we compared the average reported strength in the tactile stimulation conditions compared to the reported strength in the no tactile stimulation condition with a paired-samples sign test. Data on the second question were analyzed with a paired-samples sign test as well. In addition, with Wilcoxon one-sample signed rank tests corrected for multiple comparisons (Bonferroni) we tested whether the answers on both questions differed from zero. Effect sizes for Wilcoxon onesample signed rank tests were estimated by computing $r$ (z-score divided by the square root of the total number of observations).

To compute the strength of the direction of the sensation of rotatory self-motion, answers were transformed to a negative or to a positive value if participants answered 1 or higher and indicated that they were rotating counterclockwise or clockwise, respectively. The subsequent

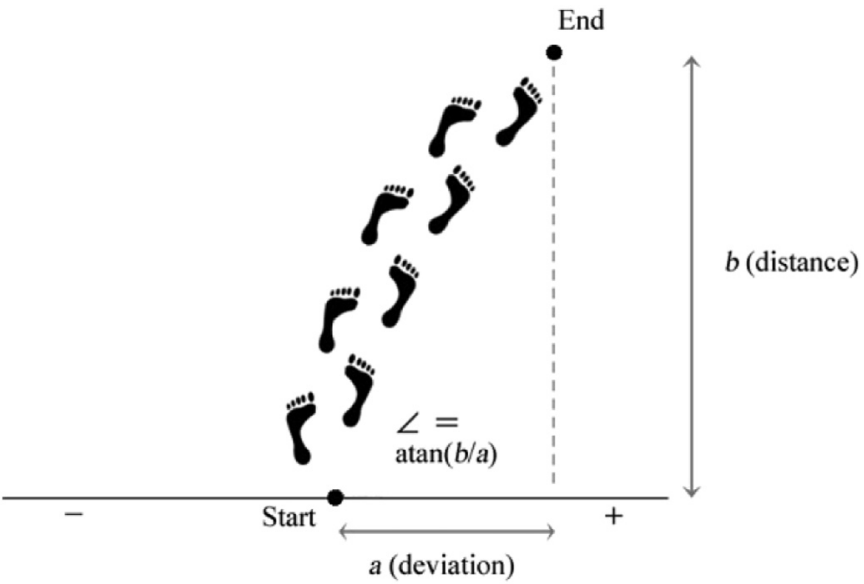

Fig. 2. The angle in which participants deviated in the walking task was computed by dividing the walked distance $(b)$ by the deviation to the left (negative number) or right (positive number) (a).

analysis was the same as the analysis (above) that did not take direction into account.

\subsubsection{Walking task}

Data of each trial in the walking task was filtered with a 50-data points moving average. The (non-absolute) angle in which participants deviated from straight ahead was computed for each trial, using the walking trajectory's endpoint distance and deviation (Fig. 2). A negative angle represented a deviation of the participant to the left and a positive angle represented a deviation to the right. Absolute angles were computed as well to examine possible non-systematic effects of tactile stimulation on participants' walking trajectory.

The walking tasks' outcome measures were analyzed with Friedman tests, Kendall's $W$ tests, and post-hoc multiple comparisons following the stepwise step-down method. In addition, difference scores between the average of the tactile stimulation conditions and the no tactile stimulation condition were computed for the outcome measures. Each of these difference scores were entered in a Spearman correlation analysis with the difference scores for subjectively reported circular vection on the first question.

\subsubsection{Balance task}

Each trial of the balance task was filtered with a first-order low-pass filter with $\tau=0.054$. For each trial, $20 \mathrm{~s}$ of data were selected. For trials in which tactile stimulation was given, data was selected from when the stimulation started. For trials without tactile stimulation, data was selected from $13.87 \mathrm{~s}$ after starting the data collection. Several outcome measures were computed for each individual trial on the selected data, namely: the slope of the data in the medio-lateral dimension; the standard error of the mean (SEM) in the medio-lateral dimension; the SEM in the anterior-posterior dimension; and the sway velocity (i.e. distance between data points divided by $20 \mathrm{~s}$ ).

The balance tasks' outcome measures were analyzed with Friedman tests, Kendall's $W$ tests and post-hoc multiple comparisons following the stepwise step-down method. In addition, difference scores between the average of the tactile stimulation conditions and the no tactile stimulation condition were computed for the outcome measures. Each of these difference scores were entered in a Spearman correlation analysis with the difference scores for subjectively reported circular vection on the first question.

\subsubsection{Exploratory analyses}

As an exploratory examination, analyses of the walking and balance data were also performed separately for the group of participants who reported circular vection with tactile stimulation (i.e. participants that 
reported circular vection with a strength of $>0$ in both tactile stimulation conditions and 0 or 1 in the no-stimulation condition on the first question).

\section{Results and discussion}

Due to time limitations, 2 of the 40 included participants did not participate in the balance task. Additionally, we excluded 1 participant from the walking trajectory task and 5 participants from the balance task, as more than half of the trials in a condition of these participants had to be excluded. (In total, 13 out of 600 and 36 out of 456 trials had to be excluded from the walking and balance tasks respectively because of problems regarding the connection with the tactile belt. In 2 of the excluded trials from the walking task, participants started moving too early.) Thus, 40 participants were included in analysis of the subjective task, 39 in the walking task, and 33 in the balance task.

\subsection{Subjective task}

Regarding the strength of rotatory self-motion as reported on question 1 ("How strongly do you perceive that you are rotating?"), ratings did not differ significantly between the clockwise (Mean $=0.68$, Median $=0, \quad S D=1.02, \quad$ Range $=0-4), \quad$ counterclockwise $($ Mean $=0.68$, Median $=0, S D=0.92$, Range $=0-3)$, and no tactile stimulation (Mean $=0.50$, Median $=0, S D=0.82, \quad$ Range $=0-3$ ) conditions, $\chi^{2}(2)=1.98, p=0.374, W=0.03$. Additionally, the averages of the tactile stimulation conditions (Mean $=0.68$, Median $=0, S D=0.92$, Range $=0-3.5$ ) did not differ significantly from the no tactile stimulation condition, $p=0.115$. All three conditions did differ from zero, all $Z \geq 3.44, p \leq 0.003, r \geq 0.54$.

Ratings on question 2 ("How strongly do you perceive that you are rotating with reference to the external environment as opposed to perceiving something moving around your body?") did not differ significantly between the clockwise (Mean $=0.58, \quad$ Median $=0$, $S D=0.90, \quad$ Range $=0-3) \quad$ and counterclockwise conditions $($ Mean $=0.48$, Median $=0, S D=0.82$, Range $=0-3), p=0.289$. Both conditions differed from zero, both $Z \geq 3.13, p \leq 0.004, r \geq 0.49$.

The results for the strength of self-reported rotatory self-motion (irrespective of the indicated direction) suggested that absolute rotatory self-motion was perceived in all three conditions (even in the condition without stimulation) with effect sizes being considered as large. However, these effects appeared to be nonspecific as there was no difference in perceived rotatory self-motion between the three conditions.

Regarding the transformed data based on the indicated direction of rotatory self-motion, ratings on question 1 differed between the clockwise $($ Mean $=0.48$, Median $=0, S D=1.13$ Range $=-2-4$ ), counterclockwise $\quad($ Mean $=-0.48, \quad$ Median $=0, \quad S D=1.01$, Range $=-3-1$ ), and no tactile stimulation (Mean $=0.00$, Median $=0$, $S D=0.91, \quad$ Range $=-3-3$ ) conditions, $\chi^{2}(2)=8.95, \quad p=0.011$, $W=0.11$. Subsequent stepwise step-down analysis showed that the clockwise and counterclockwise conditions belonged to different homogenous subsets. Additionally, the averages of the tactile stimulation conditions (Mean $=0.00$, Median $=0, S D=0.51$, Range $=-2.5$ 1) did not differ significantly from the no tactile stimulation condition, $p=1$. The tactile stimulation conditions differed from zero, $Z=2.49$, $p=0.039, r=0.39$ for the clockwise and $Z=-2.74, p=0.018$, $r=-0.43$ for the counterclockwise condition. The no stimulation condition did not differ significantly from zero, $Z=0, p=1, r=0$.

Ratings on question 2 differed between the clockwise $(M=0.32$, $S D=1.02)$ and counterclockwise conditions $(M=-0.40, S D=0.84)$, $p=0.007$. The counterclockwise tactile stimulation condition differed from zero, $Z=-2.72, p=0.012, r=-0.43$. Yet, the clockwise tactile stimulation condition did not, $Z=1.98, p=0.094, r=0.31$.

The results on the transformed subjective data demonstrated a significant effect of condition being mainly caused by the difference between the two tactile stimulation conditions. Rotatory self-motion was perceived in a clockwise direction in the clockwise stimulation condition and in a counterclockwise direction in the counterclockwise stimulation condition with medium effect sizes. No rotatory self-motion in a specific direction was perceived in the no stimulation condition, suggesting that tactile stimulation might have had a specific effect on self-reported rotatory self-motion.

\subsection{Walking and balance task - whole group level}

Analyses of the walking task data of all 39 participants, did not demonstrate a significant effect of tactile stimulation on the non-absolute or on the absolute angle, $\chi^{2}(2)=1.85, p=0.397, W=0.02$ and $\chi^{2}(2)=0.67, p=0.717, W=0.01$ respectively. Regarding the difference scores analyses, no significant correlations with subjective rotatory self-motion difference scores (non-transformed or transformed based on indicated direction) were demonstrated, not for the non-absolute or the absolute angle, all $r_{s} \leq \mp 0.12, p \geq 0.462$.

There were no significant effects of tactile stimulation on any of the outcome measures of the balance task (slope in the medio-lateral dimension, SEM in the medio-lateral dimension, SEM in the anteriorposterior dimension, and sway velocity, all $\chi^{2} \leq 2.45, p \geq 0.294$, $W \leq 04$ ) when considering the data of all 33 participants. Regarding the difference scores analyses, no significant correlations with subjective rotatory self-motion (non-transformed or transformed based on indicated direction) were demonstrated, all $r_{s} \leq \mp 0.27, p \geq 0.123$.

At the whole group level, no effects of tactile stimulation were found on walking and balance, with low concordance between participants and small correlation coefficients. Yet, this might be expected, as most participants did not report circular vection with tactile stimulation. Nine out of forty participants scored $>0$ or $<0$ in both tactile stimulation conditions and 0,1 or -1 in the no-stimulation condition on the first question (as regards the transformed data based on the indicated direction of rotatory self-motion). These nine participants were classified as participants who reported circular vection with tactile stimulation. Their average reported strength of rotatory self-motion was $1.56(S D=0.88), 1.78(S D=0.67)$, and $0.44(S D=0.53)$ for the counterclockwise, clockwise, and no tactile stimulation conditions respectively. Their average transformed ratings were $-1.33(S D=1.23)$, $1.11(S D=1.62)$, and $0.22(S D=0.44)$ for the counterclockwise, clockwise, and no tactile stimulation conditions respectively. This indicated that the subset generally experienced rotatory self-motion in the same direction as the movement of the tactile stimulation. Eight of the nine participants showed internal consistency in their answers, i.e. they reported rotation in a different direction in the clockwise and counterclockwise tactile stimulation conditions. As a comparison, only 3 out of the 31 participants that were regarded as not reporting circular vection showed internal consistency in their answers. This suggested that subjective reports in the subgroup were not random. As an exploratory examination of the effects of tactile stimulation in the subgroup of participants reporting circular vection, the analyses on the walking $(N=9)$ and balance $(N=7)$ task as reported above were also performed separately for these participants. (The discrepancy in the number of participants reporting circular vection for the walking and balance data is caused by the fact that 2 participants that reported circular vection had to be excluded from the balance data [for more information see Section 3 above]).

\subsection{Walking and balance task - participants that reported circular vection (exploratory)}

Regarding the walking task data, the results did not demonstrate significant effects on the non-absolute, nor absolute angle, for the group of nine participants that reported circular vection with tactile stimulation, $\quad \chi^{2}(2)=1.56, \quad p=0.459, \quad W=0.09 \quad$ and $\quad \chi^{2}(2)=2.00$, $p=0.368, \quad W=0.11$ respectively. Additionally, regarding the 
difference scores analyses, no significant correlations with subjective rotatory self-motion (non-transformed or transformed based on indicated direction) were demonstrated, all $r_{s} \leq \mp 0.59, p \geq 0.096$.

Regarding the balance task, no significant effects of tactile stimulation on any of the outcome measures of the balance task for the participants that reported vection $\left(\mathrm{N}=7\right.$, all $\chi^{2} \leq 2.00, p \geq 0.368$, $W \leq 0.14$ ) were obtained. Yet, correlation analyses of the difference scores for this group of participants revealed a significant negative relationship between sway velocity and non-transformed (irrespective of rotation direction) subjective rotatory self-motion, $r_{s}=0.84, p=0.019$ and a significant positive relationship between SEM in the medio-lateral dimension and transformed (based on the indicated direction) subjective rotatory self-motion, $r_{s}=0.81, p=0.028$. No other correlations were significant, all other $r_{s} \leq \mp 0.51, p \geq 0.240$.

For participants that reported circular vection with tactile stimulation no significant effects were found for the walking task data, although there was small concordance between participants for the effect of tactile stimulation on the absolute angles and several correlations with the subjective data were large. Additionally, no significant effects of tactile stimulation were found on the balance data, although concordance between participants was of small size for several effects. Yet, as sway velocity and SEM in the medio-lateral dimension highly correlated with subjective ratings of rotatory self-motion, it appeared that tactile stimulation might have been able to induce rotatory vection in participants that reported circular vection.

\section{General discussion}

The aim of the current study was to investigate whether tactile stimulation encircling the waist could induce circular vection around the body's yaw axis and to examine whether this type of stimulation would influence participants' walking trajectory and balance. It was hypothesized that the tactile stimulation would lead to self-reported circular vection in the opposite direction of the tactile stimulation and a shift in participants' walking trajectory and balance in the same direction as the tactile stimulation.

Tactile stimulation encircling the waist led to weak self-reported circular vection in a subset of participants when the indicated direction of subjective rotatory self-motion was taken into account. Participants in this subset ( 9 out of 40 participants; $22.5 \%$ ) reported circular vection with both directions of tactile stimulation (on the first question) and were able to indicate the direction of the experienced rotation. Eight of these nine participants showed internal consistency in their answers, i.e. they reported rotation in a different direction in the clockwise and counterclockwise tactile stimulation conditions. Contrary to our hypothesis, in this subset of participants, circular vection was on average experienced in the same direction as the tactile stimulation. At the whole-group level, tactile stimulation did not have an effect on participants' walking trajectory and balance. Yet, in the subset that reported circular vection the ratings (i.e. perceived strength) of rotatory selfmotion, irrespective of the indicated direction of self-rotation, correlated with sway velocity. Additionally, the ratings of rotatory self-motion that were transformed based on the indicated direction of self-rotation correlated with the SEM of sway in the medio-lateral dimension. As, for the subset of participants that reported circular vection, the subjective ratings were associated with two implicit and, importantly, objective measures of balance, the results suggest that tactile stimulation encircling the waist was able to induce circular vection in these participants. Below we will discuss these findings and will suggest that the area of stimulation and cognitive factors may be of importance in inducing vection with tactile stimulation.

Why does it appear that weak vection was induced in only a subset of participants, and why was it experienced in the direction of the tactile stimulation? To start with, tactile stimulation might not have been strong enough; a relatively small area of the torso was stimulated with a single ring of 13 tactors generating a $158 \mathrm{~Hz}$ oscillation. A stronger stimulation (covering a larger area of the body) might have increased the occurrence and compellingness of vection. In addition to the possibility that the stimulation might have been too weak, our tactile stimulation might have been perceived as an object and not as an earth-fixed background, which might have made our stimulation less effective in inducing circular vection and/or made the perceived direction of vection less consistent. Seno et al. (2009) state that figureground (object-background) segmentation is an important factor in inducing vection in which the background dominantly induces vection and the object is being less able to induce vection. In addition, Holten, van der Smagt, Verstraten, and Donker (2016) have demonstrated that low contrast moving visual stimuli that induce translational vection can induce postural sway in the opposite direction of the moving visual stimuli (depending on movement speed). Therefore, tactile stimulation covering the whole body might be more effective in inducing vection and might also induce vection in the opposite direction of the tactile stimulation.

In addition, tactile stimulation covering a larger area of the body might induce a stronger bottom-up effect. Visual vection is for a large part driven by bottom-up factors (i.e. physical stimulus properties, for example contrast and field of view), however top-down factors (i.e. cognitive factors, for example expectations and interpretations) are also able to influence visual vection (Riecke et al., 2005). Recent research even demonstrates that the motion-aftereffect, induced by moving visual stimuli, can elicit postural sway, which suggests that vection can also be internally driven (Holten, van der Smagt, Donker, and Verstraten, 2014). For tactile stimulation to induce vection top-down factors might be necessary as well, while for visual stimulation to induce vection bottom-up factors appear to be sufficient. Additionally, tactile information during everyday interactions is less likely than for example visual, kinesthetic and vestibular information to provide information about the relative position and movement of the perceiver and the environment. Under normal circumstances, it is therefore assumed that tactile information has a lower weight in determining selfmotion. Tactile information might still suggest that movement may be occurring, especially when its weight is increased, and might only be perceived as (illusory) self-movement due to top-down factors like expectations and interpretation of the stimulation (Nordahl et al., 2012; van Erp, 2007). Possibly, tactile vection might occur in a more bottomup matter after participants are taught the association between selfmotion and a tactile stimulation. An association like this was actually learned in the earlier study in which tactile circular vection was anecdotally reported (Bos et al., 2005; van Erp et al., 2006).

Why was walking not associated with subjective reports of rotatory self-motion in participants that self-reported circular vection while balance was? As vection and shifts in body displacements are highly related (e.g. Fushiki et al., 2005 (see however Guerraz and Bronstein, 2008)) and as deviations in walking trajectories have been reported with stimulation of the vestibular system (Bent et al., 2000;) it was expected that walking would be associated with subjective self-motion at least for participants that self-reported circular vection. However, as effects of vection on balance have been reported in more studies than effects on walking (Al'tman et al., 2005; Bronstein and Buckwell, 1997; Fushiki et al., 2005; Kapteyn and Bles, 1977; Reason et al., 1981; Soames and Raper, 1992; Tanaka et al., 2001), it might be the case that balance is more sensitive to self-motion illusions than walking. Indeed, concordance between participants for the walking task reached values that are being considered as small and several correlations of walking with subjective data were large, suggesting that tactile stimulation might have had a (small) effect on walking which might be statistically nonsignificant due to sample sizes being not large enough. With a tactile stimulation inducing a stronger (bottom-up) effect, or including a larger sample, participants' walking trajectory might possibly be affected as well.

Albeit only a few small effects were demonstrated in a small subset of participants, the fact that objective outcome measures correlated 
with subjective reports of vection indicates that tactile stimulation encircling the waist might be able to induce circular vection. Yet, future studies are required to further establish and examine the effects that were demonstrated in our study.

\section{Conflict of interests}

The authors declared no potential conflicts of interest with respect to research, authorship, and/or publication of this article.

\section{Acknowledgements}

The authors would like to thank Rob van de Pijpekamp for technical assistance. This research did not receive any specific grant from funding agencies in the public, commercial, or not-for-profit sectors.

\section{References}

Al'tman, Y. A., Varyagina, O. V., \& Levik, Y. S. (2005). The effects of moving sound images on postural responses and the head rotation illusion in humans. Neuroscience and Behavioral Physiology, 35(1), 103-106.

Andersen, G. J. (1986). Perception of self-motion: Psychophysical and computational approaches. Psychological Bulletin, 99(1), 52-65.

Bent, L. R., McFadyen, B. J., French Merkley, V., Kennedy, P. M., \& Inglis, J. T. (2000) Magnitude effects of galvanic vestibular stimulation on the trajectory of human gait. Neuroscience Letters, 279(3), 157-160.

Bos, J. E., van Erp, J., Groen, E. L., \& van Veen, H. J. (2005). Vestibulo-tactile interactions regarding motion perception and eye movements in yaw. Journal of Vestibular Research, 15(3), 149-160.

Bronstein, A. M., \& Buckwell, D. (1997). Automatic control of postural sway by visual motion parallax. Experimental Brain Research, 113(2), 243-248.

Cauquil, A. S., Martinez, P., Ouaknine, M., \& Tardy-Gervet, M. F. (2000). Orientation of the body response to galvanic stimulation as a function of the inter-vestibular imbalance. Experimental Brain Research, 133(4), 501-505.

Field, A. (2009). Discovering statistics using SPSS (3rd ed.). London: SAGE.

Fitzpatrick, R., Burke, D., \& Gandevia, S. C. (1994). Task-dependent reflex responses and movement illusions evoked by galvanic vestibular stimulation in standing humans. The Journal of Physiology, 478(2), 363-372.

Fushiki, H., Kobayashi, K., Asai, M., \& Watanabe, Y. (2005). Influence of visually induced self-motion on postural stability. Acta Oto-Laryngologica, 125(1), 60-64.

Guerraz, M., \& Bronstein, A. M. (2008). Mechanisms underlying visually induced body sway. Neuroscience Letters, 443(1), 12-16.

Holten, V., van der Smagt, M. J., Donker, S. F., \& Verstraten, F. A. J. (2014). Illusory motion of the motion aftereffect induces postural sway. Psychological Science, 25(9), 1831-1834.

Holten, V., van der Smagt, M. J., Verstraten, F. A. J., \& Donker, S. F. (2016). Interaction effects of visual stimulus speed and contrast on postural sway. Experimental Brain Research, 234, 113-124.

Kapteyn, T. S., \& Bles, W. (1977). Circular vection and human posture. III. Relation between the reactions to various stimuli. Agressologie, 18(6), 335-339.

Lepecq, J. C., De Waele, C., Mertz-Josse, S., Teyssedre, C., Huy, P. T. B., Baudonniere, P. M., \& Vidal, P. P. (2006). Galvanic vestibular stimulation modifies vection paths in healthy subjects. Journal of Neurophysiology, 95(5), 3199-3207.
Lestienne, F., Soechting, J., \& Berthoz, A. (1977). Postural readjustments induced by linear motion of visual scenes. Experimental Brain Research, 28(3-4), 363-384.

Nakamura, S., \& Shimojo, S. (2000). A slowly moving foreground can capture an observer's self-motion-A report of a new motion illusion: Inverted vection. Vision Research, 40(21), 2915-2923.

Nakamura, S., \& Shimojo, S. (2003). Sustained deviation of gaze direction can affect "inverted vection" induced by the foreground motion. Vision Research, 43(7), 745-749.

Nilsson, N. C., Nordahl, R., Sikström, E., Turchet, L., \& Serafin, S. (2012). Haptically induced illusory self-motion and the influence of context of motion. In P. Isokoski, \& J. Springare (Eds.). Haptics: Perception, devices, mobility, and communication (pp. 349360). Berlin, Heidelberg: Springer.

Nordahl, R., Nilsson, N. C., Turchet, L., \& Serafin, S. (2012). Vertical illusory self-motion through haptic stimulation of the feet. Perceptual illusions in virtual environments (pp. 21-26). .

Reason, J., Wagner, H., \& Dewhurst, D. (1981). A visually-driven postural after-effect Acta Psychologica, 48(1), 241-251.

Riecke, B. E., Feuereissen, D., \& Rieser, J. J. (2008). Auditory self-motion illusions (circular vection) can be facilitated by vibrations and the potential for actual motion. Proceedings of the 5th symposium on Applied perception in graphics and visualization (pp. 147-154). ACM.

Riecke, B. E., Schulte-Pelkum, J., Caniard, F., \& Bulthoff, H. H. (2005). Towards lean and elegant self-motion simulation in virtual reality. Virtual reality proceedings (pp. 131138).

Riecke, B. E., Väljamäe, A., \& Schulte-Pelkum, J. (2009). Moving sounds enhance the visually-induced self-motion illusion (circular vection) in virtual reality. ACM Transactions on Applied Perception (TAP), 6(2), 7.

Roll, R., Kavounoudias, A., \& Roll, J. P. (2002). Cutaneous afferents from human plantar sole contribute to body posture awareness. Neuroreport, 13(15), 1957-1961.

Seno, T., Ito, H., \& Sunaga, S. (2009). The object and background hypothesis for vection. Vision Research, 49(24), 2973-2982.

Seno, T., Ogawa, M., Ito, H., \& Sunaga, S. (2011). Consistent air flow to the face facilitates vection. Perception, 40(10), 1237-1240.

Soames, R. W., \& Raper, S. A. (1992). The influence of moving auditory fields on postural sway behaviour in man. European Journal of Applied Physiology and Occupational Physiology, 65(3), 241-245.

Tanaka, T., Kojima, S., Takeda, H., Ino, S., \& Ifukube, T. (2001). The influence of moving auditory stimuli on standing balance in healthy young adults and the elderly. Ergonomics, 44(15), 1403-1412.

Väljamäe, A. (2005). Self-motion and presence in the perceptual optimization of a multisensory virtual reality environment (Doctoral dissertation)Göteborg, Sweden: Chalmers University of Technology.

Väljamäe, A. (2009). Auditorily-induced illusory self-motion: A review. Brain Research Reviews, 61(2), 240-255.

van Erp, J. B. (2007). Tactile displays for navigation and orientation: perception and behaviour (Doctoral dissertation)Utrecht, The Netherlands: Utrecht University.

van Erp, J. B., Groen, E. L., Bos, J. E., \& van Veen, H. A. (2006). A tactile cockpit instrument supports the control of self-motion during spatial disorientation. Human Factors: The Journal of the Human Factors and Ergonomics Society, 48(2), 219-228.

van Erp, J. B., \& van Veen, H. A. (2006). Touch down: The effect of artificial touch cues on orientation in microgravity. Neuroscience Letters, 404(1), 78-82.

Wardman, D. L., Taylor, J. L., \& Fitzpatrick, R. C. (2003). Effects of galvanic vestibular stimulation on human posture and perception while standing. The Journal of Physiology, 551(3), 1033-1042.

Wright, W. G., DiZio, P., \& Lackner, J. R. (2006). Perceived self-motion in two visual contexts: Dissociable mechanisms underlie perception. Journal of Vestibular Research, 16(1), 23-28. 